\title{
Tensor products of Archimedean partially ordered vector spaces
}

\author{
Onno van Gaans • Anke Kalauch
}

Received: 31 January 2010 / Accepted: 13 September 2010 / Published online: 1 October 2010 (C) The Author(s) 2010. This article is published with open access at Springerlink.com

\begin{abstract}
We study the tensor product of two directed Archimedean partially ordered vector spaces $X$ and $Y$ by means of Riesz completions. With the aid of the Fremlin tensor product of the Riesz completions of $X$ and $Y$ we show that the projective cone in $X \otimes Y$ is contained in an Archimedean cone. The smallest Archimedean cone containing the projective cone satisfies an appropriate universal mapping property.
\end{abstract}

Keywords Archimedean partially ordered vector space - Fremlin tensor product . Projective cone $\cdot$ Riesz completion · Tensor product

\section{Mathematics Subject Classification (2000) $\quad 06 \mathrm{~F} 20 \cdot 46 \mathrm{~A} 40$}

\section{Introduction}

Tensor products of function spaces are common objects in functional analytic considerations. These tensor products often have a natural lattice order, just as their components. D. H. Fremlin has studied a general construction of a tensor product of Riesz spaces (vector lattices) which is compatible with the natural tensor products of function spaces. It turns out that such a construction is quite involved. In his seminal paper [7], Fremlin views the algebraic vector space tensor product of two function spaces as a space of functions on the Cartesian product of the underlying spaces. Then he

O. van Gaans ( $\square)$

Mathematical Institute, Leiden University, P.O. Box 9512,

2300 RA Leiden, The Netherlands

e-mail: vangaans@math.leidenuniv.nl

\section{A. Kalauch}

Institut für Analysis, Fachrichtung Mathematik, TU Dresden, 01062 Dresden, Germany 
endows this space with the induced order. As this space is in general not a Riesz space, he considers the Riesz subspace generated by the vector space tensor product. Fremlin succeeds with a similar construction to make a Riesz space tensor product for arbitrary Archimedean Riesz spaces. His construction uses representations of Archimedean Riesz spaces with order units as subspaces of spaces of continuous functions.

The main point where the representation theory in Fremlin's construction is used, is the construction of the ambient space of which the vector space tensor product is a subspace. This ambient space yields the induced order on the vector space tensor product and it yields the completion to a Riesz space. The main difficulties in finding an intrinsic construction are the definition of the appropriate order on the vector space tensor product and the completion of this partially ordered vector space to a Riesz space.

An alternative construction of the Fremlin tensor product is provided by the theory of tensor products of $\ell$-groups in $[5,10]$.

Grobler and Labuschagne [8] give an intrinsic construction of the Fremlin tensor product. They begin with the projective cone in the vector space tensor product, which is the cone generated by the elementary tensors of positive elements. They show that the relative uniform closure of the projective cone induces the appropriate order on the tensor product. The Fremlin tensor product is then obtained by means of Dedekind completions. On their way they also construct the tensor product of directed Archimedean partially ordered vector spaces with the Riesz decomposition property. In the latter case the order on the tensor product is required to satisfy a universal mapping property (see Definition 4.1). One of the major steps in the construction of Grobler and Labuschagne where the Riesz decomposition property is used is the proof that the relative uniform closure of the projective cone is again a cone and not just a wedge.

It is our intention to study tensor products of partially ordered vector spaces by means of Riesz completions. The notion of pre-Riesz space and Riesz completion have been introduced by van Haandel in [14]. We consider two directed Archimedean partially ordered vector spaces $X$ and $Y$ and show that the relative uniform closure of the projective cone in the vector space tensor product $X \otimes Y$ is a cone with the desired universal mapping property. The proof uses the Riesz completions of $X$ and $Y$ and their Fremlin tensor product. Our result extends [8, Theorem 2.5] since we do not need the Riesz decomposition property.

Section 2 recalls the vector space tensor product and the projective cone. In Sect. 3 we recall the required terminology on Riesz completions and present Fremlin's tensor product as Riesz completion. In Sect. 4 we use Riesz completions to construct the appropriate cone in the tensor product of directed Archimedean partially ordered vector spaces.

\section{The tensor product and the projective cone}

Let us recall the definitions and main results of the vector space tensor product and the projective cone. The standard facts are given without proof or reference. For the reader's convenience complete proofs are provided in [13]. 
Definition 2.1 Let $X$ and $Y$ be (real) vector spaces. A pair $(T, \tau)$ is called a tensor product of $X$ and $Y$ provided

(1) $T$ is a vector space and $\tau: X \times Y \rightarrow T$ is a bilinear map; and

(2) if $S$ is a vector space and $\sigma: X \times Y \rightarrow S$ is a bilinear map, then there is a unique linear map $\sigma^{*}: T \rightarrow S$ such that $\sigma(x, y)=\sigma^{*}(\tau(x, y))$ for all $x \in X$ and $y \in Y$.

Remark 2.2 If $(T, \tau)$ is a tensor product of $X$ and $Y$, then $\{\tau(x, y): x \in X, y \in Y\}$ spans $T$. Indeed, for

$$
S=\operatorname{span}\{\tau(x, y): x \in X, y \in Y\}
$$

and $\sigma=\tau$ there is a linear map $\sigma^{*}: T \rightarrow S$ such that $\tau(x, y)=\sigma^{*}(\tau(x, y))$ for all $x \in X$ and $y \in Y$. Suppose that $T \backslash S \neq \varnothing$. By choosing first a Hamel basis of $S$ and then extending it to a basis of $T$, one can construct a linear map $\phi: T \rightarrow \mathbb{R}$ with $\phi \neq 0$ and $\phi=0$ on $S$. Choose $s \in S$ with $s \neq 0$ and define $\sigma_{2}^{*}: T \rightarrow S$ by $\sigma_{2}^{*}(t)=$ $\sigma^{*}(t)+\phi(t) s$. Then $\sigma_{2}^{*}=\sigma^{*}$ on $S$, in particular $\tau(x, y)=\sigma_{2}^{*}(\tau(x, y))$ for all $x \in X$ and $y \in Y$, and for $u \in T$ with $\phi(u) \neq 0$ one has $\sigma_{2}^{*}(u)=\sigma^{*}(u)+\phi(u) s \neq \sigma^{*}(u)$, which contradicts the uniqueness of $\sigma^{*}$.

Theorem 2.3 Let $X$ and $Y$ be vector spaces.

(a) There exists a tensor product $(T, \tau)$ of $X$ and $Y$.

(b) If $(\hat{T}, \hat{\tau})$ is another tensor product of $X$ and $Y$, then there is a linear bijection

$$
\tau^{*}: \hat{T} \rightarrow T
$$

such that $\tau(x, y)=\tau^{*}(\hat{\tau}(x, y))$ for all $x \in X, y \in Y$.

It follows from Theorem 2.3 that there exists an essentially unique tensor product $(T, \tau)$ of $X$ and $Y$. We denote it as usual by $X \otimes Y$, and for $x \in X$ and $y \in Y$ we use the notation $x \otimes y=\tau(x, y)$.

The next lemma will be needed in the sequel to relate the tensor product of pre-Riesz spaces and the tensor product of their Riesz completions.

Lemma 2.4 Let $X, Y, U$ and $V$ be vector spaces and let $\rho_{X}: X \rightarrow U$ and $\rho_{Y}: Y \rightarrow V$ be linear injections. Let

$$
\rho(x, y):=\rho_{X}(x) \otimes \rho_{Y}(y), \quad x \in X, y \in Y .
$$

Then the unique linear map $\rho^{*}: X \otimes Y \rightarrow U \otimes V$ satisfying $\rho^{*}(x \otimes y)=\rho(x, y)$ for all $x \in X$ and $y \in Y$ is injective.

Proof Let $w, z \in X \otimes Y$ be such that $\rho^{*}(w)=\rho^{*}(z)$. Due to Remark 2.2, there are $x_{i} \in X, y_{i} \in Y$, and $\alpha_{i} \in \mathbb{R}, i \in\{1, \ldots, n\}$ such that

$$
z=\sum_{i=1}^{n} \alpha_{i} x_{i} \otimes y_{i}
$$


Choose a Hamel basis $\left(e_{i}\right)_{i \in I}$ of $\rho_{X}(X)$ and extend it to a Hamel basis $\left(e_{i}\right)_{i \in I^{\prime}}$ of $U$ and choose a Hamel basis $\left(f_{j}\right)_{j \in J}$ of $\rho_{Y}(Y)$ and extend it to a Hamel basis $\left(f_{j}\right)_{j \in J^{\prime}}$ of $V$. Define $\eta: U \times V \rightarrow X \otimes Y$ by

$$
\eta\left(\sum_{i \in I^{\prime}} \lambda_{i} e_{i}, \sum_{j \in J^{\prime}} \mu_{j} f_{j}\right):=\sum_{i \in I} \sum_{j \in J} \lambda_{i} \mu_{j} \rho_{X}^{-1}\left(e_{i}\right) \otimes \rho_{Y}^{-1}\left(f_{j}\right)
$$

Then $\eta$ is bilinear and $\eta\left(\rho_{X}(x), \rho_{Y}(y)\right)=x \otimes y$ for all $x \in X$ and $y \in Y$. Hence there is a unique linear map $\eta^{*}: U \otimes V \rightarrow X \otimes Y$ such that $\eta^{*}(u \otimes v)=\eta(u, v)$ for all $u \in U, v \in V$. In particular, $\eta^{*}\left(\rho_{X}(x) \otimes \rho_{Y}(y)\right)=x \otimes y$ for all $x \in X$ and $y \in Y$. We obtain that

$$
\begin{aligned}
\eta^{*}\left(\rho^{*}(z)\right) & =\sum_{i=1}^{n} \alpha_{i} \eta^{*}\left(\rho^{*}\left(x_{i} \otimes y_{i}\right)\right)=\sum_{i=1}^{n} \alpha_{i} \eta^{*}\left(\rho\left(x_{i}, y_{i}\right)\right) \\
& =\sum_{i=1}^{n} \alpha_{i} \eta^{*}\left(\rho_{X}\left(x_{i}\right) \otimes \rho_{Y}\left(y_{i}\right)\right)=\sum_{i=1}^{n} \alpha_{i} x_{i} \otimes y_{i}=z .
\end{aligned}
$$

Similarly, $\eta^{*}\left(\rho^{*}(w)\right)=w$. From $\rho^{*}(z)=\rho^{*}(w)$ it follows that $z=w$.

Before we consider the projective cone, we recall some terminology on partially ordered vector spaces.

By $X$ we denote a real vector space and by $K$ a cone in $X$, that is, $K$ is a wedge $(x, y \in K, \lambda, \mu \geq 0$ imply $\lambda x+\mu y \in K)$ and $K \cap(-K)=\{0\}$. In $X$ a partial order is introduced by defining $y \geq x$ if and only if $y-x \in K$. Denote for a subset $M \subseteq X$ the set of all upper bounds by

$$
M^{u}=\{x \in X: x \geq m \text { for all } m \in M\} .
$$

The space $(X, K)$ is called Archimedean if for every $x, y \in X$ with $n x \leq y$ for all $n \in \mathbb{N} \cup\{0\}$ one has $x \leq 0$. A set $M \subseteq X$ is called directed if for every $x, y \in M$ there is an element $z \in M$ such that $z \geq x$ and $z \geq y . X$ is directed if and only if the cone $K$ is generating in $X$, that is, $X=K-K$. $X$ has the Riesz decomposition property if for every $y, x_{1}, x_{2} \in K$ with $y \leq x_{1}+x_{2}$ there exist $y_{1}, y_{2} \in K$ such that $y=y_{1}+y_{2}$ and $y_{1} \leq x_{1}, y_{2} \leq x_{2}$. For standard notions in the case that $X$ is a Riesz space, see [1].

Let $\left(X, K_{X}\right)$ and $\left(Y, K_{Y}\right)$ be partially ordered vector spaces. In the vector space tensor product $T=X \otimes Y$ one defines (see, e.g., [8])

$$
K_{T}:=\left\{\sum_{i=1}^{n} \alpha_{i} x_{i} \otimes y_{i}: x_{i} \in K_{X}, y_{i} \in K_{Y}, \alpha_{i} \in \mathbb{R}^{+}, n \in \mathbb{N}\right\}
$$

Theorem $2.5\left(T, K_{T}\right)$ is a partially ordered vector space. If $X$ and $Y$ are directed partially ordered vector spaces, then $K_{T}$ is generating in $T$. 
A proof of Theorem 2.5 can be found in, e.g., [13].

$K_{T}$ is called the projective cone in $T$.

\section{Riesz completions and the Fremlin tensor product}

A partially ordered vector space $X$ is called pre-Riesz if for every $x, y, z \in X$ the inclusion $\{x+y, x+z\}^{u} \subseteq\{y, z\}^{u}$ implies $x \in K$ [14, Definition 1.1 (viii), Theorem 4.15]. Every pre-Riesz space is directed and every directed Archimedean partially ordered vector space is pre-Riesz [14]. Clearly, each Riesz space is pre-Riesz.

By a subspace of a partially ordered vector space or a Riesz space we mean an arbitrary linear subspace with the inherited order. We do not require it to be a lattice or a sublattice. We say that a subspace $X$ of a Riesz space $Y$ generates $Y$ as a Riesz space if for every $y \in Y$ there exist $a_{1}, \ldots, a_{m}, b_{1}, \ldots, b_{n} \in X$ such that $y=\bigvee_{i=1}^{m} a_{i}-\bigvee_{i=1}^{n} b_{i}$.

We call a linear subspace $D$ of a partially ordered vector space $X$ order dense in $X$ if for every $x \in X$ we have $x=\inf \{y \in D: y \geq x\}$, that is, each $x$ is the greatest lower bound of the set $\{y \in D: y \geq x\}$ in $X$.

Recall that a linear map $i: X \rightarrow Y$, where $X$ and $Y$ are partially ordered vector spaces, is called bipositive if for every $x \in X$ one has $i(x) \geq 0$ if and only if $x \geq 0$. An embedding map is required to be linear and bipositive, which implies injectivity.

Let $X$ be a partially ordered vector space. According to van Haandel [14, Corollaries 4.9-11 and Theorems 3.5, 3.7, 4.13] the following statements are equivalent:

(i) $X$ is pre-Riesz.

(ii) There exist a Riesz space $Y$ and a bipositive linear map $i: X \rightarrow Y$ such that $i(X)$ is order dense in $Y$.

(iii) There exist a Riesz space $Y$ and a bipositive linear map $i: X \rightarrow Y$ such that $i(X)$ is order dense in $Y$ and generates $Y$ as a Riesz space.

All spaces $Y$ as in (iii) are isomorphic as Riesz spaces. A pair $(Y, i)$ as in (iii) is called a Riesz completion of $X$. As it is unique up to isomorphism we will speak of the Riesz completion of $X$ and denote it by $X^{\rho}$. If $X$ is Archimedean, then its Riesz completion coincides with its enveloping Riesz space, as defined below Theorem 2.9 in [4].

Van Haandel also introduces a notion of homomorphism adapted to the concept of pre-Riesz spaces. Let $X$ and $Y$ be directed partially ordered vector spaces. A linear map $h: X \rightarrow Y$ is called a Riesz* homomorphism if for any $a, b \in X$ and for every lower bound $x$ of $\{a, b\}^{u}$ in $X$ one has that $h(x)$ is a lower bound of $\{h(a), h(b)\}^{u}$ in $Y$ (see [14, Definition 5.1]). If $X$ and $Y$ are Riesz spaces, then $h: X \rightarrow Y$ is a Riesz* homomorphism if and only if it is a Riesz homomorphism. If $X$ and $Y$ are pre-Riesz spaces, then $h: X \rightarrow Y$ is a Riesz* homomorphism if and only if it is the restriction of a Riesz homomorphism from $X^{\rho}$ into $Y^{\rho}$. The composition of two Riesz* homomorphisms is a Riesz* homomorphism (see [14, Remarks 5.2(ii), (iii), and Theorem 5.6]).

Next we recall Fremlin's main result on the tensor product of Archimedean Riesz spaces $\left(E, E^{+}\right)$and $\left(F, F^{+}\right)$.

Theorem 3.1 [7, 4.2] Let $E$ and $F$ be Archimedean Riesz spaces. Then there is an essentially unique Archimedean Riesz space $G$ and a Riesz bimorphism $\phi: E \times F \rightarrow$ G such that 
(i) whenever $H$ is an Archimedean Riesz space and $\psi: E \times F \rightarrow H$ is a Riesz bimorphism, there is a unique Riesz homomorphism $T: G \rightarrow H$ such that $T \phi=\psi$;

(ii) $\phi$ induces an embedding of $E \otimes F$ in $G$;

(iii) $E \otimes F$ is dense in $G$ in the sense that for every $w \in G$ there exist $x_{0} \in E$ and $y_{0} \in F$ such that for every $\delta>0$ there is $a v \in E \otimes F$ such that $|w-v| \leq \delta x_{0} \otimes y_{0}$

(iv) if $w>0$ in $G$, then there exist $x \in E^{+}$and $y \in F^{+}$such that $0<x \otimes y \leq w$.

The essentially unique Archimedean Riesz space $G$ in the above theorem is called the Fremlin tensor product of $E$ and $F$ and is denoted by $E \bar{\otimes} F$. The essential uniqueness means that any Archimedean Riesz space $G$ with the properties of the theorem is Riesz isomorphic to $E \bar{\otimes} F$. Usually, one identifies the vector space tensor product $E \otimes F$ with the corresponding linear subspace of $E \bar{\otimes} F$.

The next result says that the Fremlin tensor product is the Riesz completion of the vector space tensor product equipped with the order induced by the Fremlin tensor product. This fact is contained in [5, Theorem 4]. A proof based on the density property in Theorem 3.1(iii) (see also [2]) is given in [13].

Theorem 3.2 Let $E$ and $F$ be Archimedean Riesz spaces, let $E \bar{\otimes} F$ be the Fremlin tensor product of $E$ and $F$, and let $E \otimes F$ be the linear subspace generated by all $x \otimes y, x \in E, y \in F$, endowed with the induced order. Then $E \otimes F$ is a pre-Riesz space and $E \bar{\otimes} F$ is its Riesz completion. Moreover, the inclusion map $\hat{\phi}: E \otimes F \rightarrow E \bar{\otimes} F$ is a Riesz* homomorphism.

\section{Closure of the projective cone}

Consider two Archimedean Riesz spaces $X$ and $Y$, their vector space tensor product $T=X \otimes Y$, and let $K_{T}$ be the projective cone in $X \otimes Y$ as defined in Sect. 2. Although the order induced by $K_{T}$ is natural from a construction point of view, it is often not the natural order in examples. For instance, if $X=Y=C[0,1]$, then the order induced by $K_{T}$ on $X \otimes Y$ viewed as subspace of $C([0,1] \times[0,1])$ is not the natural pointwise order on $[0,1] \times[0,1]$ (see [7, Counter example 4.7]). The advantage of Fremlin's construction via representations is that it yields the natural order in examples. The problem of the cone $K_{T}$ is that it is in general non-Archimedean and smaller than the cone of Fremlin's order. The closure of $K_{T}$ has been studied in various settings with topologies or order units, see $[3,6,9,11,12]$.

It turns out that the cone of Fremlin's order can be obtained from $K_{T}$ by taking a suitable Archimedean closure of $K_{T}$. Grobler and Labuschagne have taken this approach in [8] to construct the cone of Fremlin's order intrinsically from $K_{T}$. They were able to extend their methods to directed Archimedean partially ordered vector spaces with the Riesz decomposition property. We will go even further and show that the Riesz decomposition property is not needed. Instead of embedding in bidual spaces we will use Riesz completions. Thus, we construct the tensor product of arbitrary directed Archimedean partially ordered vector spaces with the proper order. Our construction 
of the appropriate wedge in the tensor product is intrinsic. However, for the proof that it is in fact a cone, we need to rely on Fremlin's results.

In the remainder of this section, let $\left(X, K_{X}\right)$ and $\left(Y, K_{Y}\right)$ be two directed Archimedean partially ordered vector spaces, let $T=X \otimes Y$ be their vector space tensor product, and let $K_{T}$ be the projective cone as constructed in Sect. 2.

The next definition is taken from [8, Section 2].

Definition 4.1 A cone $K$ in $X \otimes Y$ is called an Archimedean tensor cone if $K_{T} \subseteq$ $K$ and the following universal mapping property is satisfied: For every directed Archimedean partially ordered vector space $\left(S, K_{S}\right)$ and every positive bilinear map $\sigma: X \times Y \rightarrow S$ the induced linear map $\sigma^{*}:(X \otimes Y, K) \rightarrow\left(S, K_{S}\right)$ is positive.

If an Archimedean tensor cone in $X \otimes Y$ exists, then it is unique [8, Section 2].

Let $\left(X^{\rho}, \rho_{X}\right)$ and $\left(Y^{\rho}, \rho_{Y}\right)$ be the Riesz completions of $X$ and $Y$, respectively. Then $X^{\rho}$ and $Y^{\rho}$ are Archimedean Riesz spaces. Let $X^{\rho} \bar{\otimes} Y^{\rho}$ be the Fremlin tensor product. Then there exists a Riesz bimorphism $\phi_{F}: X^{\rho} \times Y^{\rho} \rightarrow X^{\rho} \bar{\otimes} Y^{\rho}$ with the properties as in Theorem 3.1. In particular, there is a linear injection $h_{F}: X^{\rho} \otimes Y^{\rho} \rightarrow X^{\rho} \bar{\otimes} Y^{\rho}$ such that

$$
h_{F}(u \otimes v)=\phi_{F}(u, v) \text { for all } u \in X^{\rho}, v \in Y^{\rho} .
$$

Define $\rho: X \times Y \rightarrow X^{\rho} \otimes Y^{\rho}$ by

$$
\rho(x, y):=\rho_{X}(x) \otimes \rho_{Y}(y), \quad x \in X, y \in Y .
$$

Then $\rho$ is bilinear, so $\rho$ induces a unique linear map $\rho^{*}: X \otimes Y \rightarrow X^{\rho} \otimes Y^{\rho}$ such that

$$
\rho(x, y)=\rho^{*}(x \otimes y) \text { for all } x \in X, y \in Y .
$$

By Lemma 2.4, $\rho^{*}$ is injective. Hence $X \otimes Y$ is embedded into $X^{\rho} \bar{\otimes} Y^{\rho}$ by the injective linear map $h_{F} \circ \rho^{*}$. Thus the order of the Fremlin tensor product $X^{\rho} \bar{\otimes} Y^{\rho}$ induces an order on $X \otimes Y$. Define

$$
K_{F}:=\left\{w \in X \otimes Y: h_{F}\left(\rho^{*}(w)\right) \in\left(X^{\rho} \bar{\otimes} Y^{\rho}\right)^{+}\right\} .
$$

Lemma 4.2 $K_{F}$ is a cone in $X \otimes Y, K_{T} \subseteq K_{F}$, and $\left(X \otimes Y, K_{F}\right)$ is Archimedean.

Proof Since $h_{F}$ and $\rho^{*}$ are linear and injective, $K_{F}$ is a cone. For $x \in K_{X}$ and $y \in K_{Y}$, we have

$$
\begin{aligned}
h_{F}\left(\rho^{*}(x \otimes y)\right) & =h_{F}(\rho(x, y))=h_{F}\left(\rho_{X}(x) \otimes \rho_{Y}(y)\right) \\
& =\phi_{F}\left(\rho_{X}(x), \rho_{Y}(y)\right) \in\left(X^{\rho} \bar{\otimes} Y^{\rho}\right)^{+},
\end{aligned}
$$

as $\phi_{F}$ is a Riesz bimorphism and therefore positive. So $x \otimes y \in K_{F}$, which implies that $K_{T} \subseteq K_{F}$. 
If $w_{1}, w_{2} \in X \otimes Y$ are such that $w_{1}-n w_{2} \in K_{F}$ for all $n \in \mathbb{N}$, then $h_{F}\left(\rho^{*}\left(w_{1}\right)\right)-$ $n h_{F}\left(\rho^{*}\left(w_{2}\right)\right) \in\left(X^{\rho} \bar{\otimes} Y^{\rho}\right)^{+}$for all $n$. Then $-h_{F}\left(\rho^{*}\left(w_{2}\right)\right) \in\left(X^{\rho} \bar{\otimes} Y^{\rho}\right)^{+}$, as $X^{\rho} \bar{\otimes} Y^{\rho}$ is Archimedean. So $-w_{2} \in K_{F}$. Hence $\left(X \otimes Y, K_{F}\right)$ is Archimedean.

Due to Lemma 4.2, the cone $K_{T}$ is contained in an Archimedean cone, so there exists a smallest Archimedean cone $K$ in $X \otimes Y$ which contains $K_{T}$. $K$ is obtained as the intersection of all Archimedean cones that contain $K_{T}$. It turns out that $K$ is the Archimedean tensor cone in $X \otimes Y$. There is an alternative construction of the Archimedean tensor cone as the relatively uniform closure (ru-closure) of $K_{T}$, which we need in the proof.

We recall some facts about the relative uniform topology. Let $\left(S, K_{S}\right)$ be a directed partially ordered vector space. A sequence $\left(s_{n}\right)_{n}$ in $S$ is said to converge relatively uniformly to an $s \in S$, denoted by $s_{n} \rightarrow s$ (ru), if there exist an $a \in K_{S}$ and a sequence $\left(\lambda_{n}\right)_{n}$ in $\mathbb{R}^{+}$such that $\lambda_{n} \rightarrow 0$ as $n \rightarrow \infty$ and $-\lambda_{n} a \leq s_{n}-s \leq \lambda_{n} a$ for all $n$. A subset $C$ of $S$ is called ru-closed if it is closed under ru-convergence of sequences. It can be shown that there exists a vector space topology in $S$ for which the closed sets are exactly the ru-closed subsets of $S$. This topology is called the ru-topology in $S$. By the ru-closure of a set $C \subseteq S$ we mean the smallest ru-closed set in $S$ which contains $C$.

Lemma 4.3 $K_{F}$ is ru-closed in $\left(X \otimes Y, K_{T}\right)$.

Proof Let $\left(w_{n}\right)_{n}$ in $K_{F}$ and $w \in X \otimes Y$ be such that $w_{n} \rightarrow w\left(\right.$ ru). Take $a \in K_{T}$ and $\lambda_{n} \rightarrow 0$ such that we have in $K_{T}$-order for all $n$ that $-\lambda_{n} a \leq w_{n}-w \leq \lambda_{n} a$. Let $\tilde{w}:=h_{F}\left(\rho^{*}(w)\right), \tilde{w}_{n}:=h_{F}\left(\rho^{*}\left(w_{n}\right)\right), n \in \mathbb{N}$, and $\tilde{a}:=h_{F}\left(\rho^{*}(a)\right)$. Since $h_{F} \circ \rho^{*}$ is positive we obtain $-\lambda_{n} \tilde{a} \leq \tilde{w}_{n}-\tilde{w} \leq \lambda \tilde{a}$ in $\left(X^{\rho} \bar{\otimes} Y^{\rho}\right)^{+}$-order for all $n$. Then $\tilde{w} \geq \tilde{w}_{n}-\lambda_{n} \tilde{a} \geq-\lambda_{n} \tilde{a}$ for all $n$. As $\left(X^{\rho} \bar{\otimes} Y^{\rho}\right)^{+}$is Archimedean, we obtain $\tilde{w} \in\left(X^{\rho} \bar{\otimes} Y^{\rho}\right)^{+}$, so that $w \in K_{F}$.

Theorem 4.4 For a cone $K$ in $X \otimes Y$ the following four statements are equivalent:

(a) $K$ is the Archimedean tensor cone.

(b) Let $\left(S, K_{S}\right)$ be a directed Archimedean partially ordered vector space and let $\phi: X \otimes Y \rightarrow S$ be a linear map such that $\phi(w) \in K_{S}$ for all $w \in K_{T}$. Then $\phi(w) \in K_{S}$ for all $w \in K$.

(c) $K$ is the smallest Archimedean cone in $X \otimes Y$ with $K_{T} \subseteq K$.

(d) $K=\overline{K_{T}}$, where $\overline{K_{T}}$ is the ru-closure of $K_{T}$ in $\left(X \otimes Y, K_{T}\right)$.

Proof (a) $\Rightarrow$ (c): Let $L$ be an Archimedean cone in $X \otimes Y$ with $K_{T} \subseteq L$. Take $S:=X \otimes Y, K_{S}:=L$, and $\sigma(x, y):=x \otimes y, x \in X, y \in Y$. Since $\sigma$ is positive bilinear and $K$ is the Archimedean tensor cone, it follows that $\sigma^{*}:(X \otimes Y, K) \rightarrow(X \otimes Y, L)$ is positive. As $\sigma^{*}$ is the identity map on $X \otimes Y$, we obtain that $K \subseteq L$.

(c) $\Rightarrow$ (d): We first show that $K$ is ru-closed. Let $\left(w_{n}\right)_{n}$ in $K$ and $w \in X \otimes Y$ be such that $w_{n} \rightarrow w(\mathrm{ru})$. Then there are an $a \in K_{T}$ and $\lambda_{n} \rightarrow 0$ such that in $K_{T}$-order we have

$$
-\lambda_{n} a \leq w_{n}-w \leq \lambda_{n} a \text { for all } n \in \mathbb{N} .
$$


Then $w+\lambda_{n} a-w_{n} \in K_{T} \subseteq K$, so $w+\lambda_{n} a \in K$ for all $n$. As $K$ is Archimedean, we obtain that $w \in K$. Hence $K$ is ru-closed, so that $\overline{K_{T}} \subseteq K$.

Since $K_{T} \subseteq K_{F}$ and $K_{F}$ is ru-closed according to Lemma 4.3, we have $\overline{K_{T}} \subseteq K_{F}$. As $K_{F}$ is a cone by Lemma 4.2 and $\overline{K_{T}}$ is a wedge, it follows that $\overline{K_{T}}$ is a cone.

Next we show that $\overline{K_{T}}$ is Archimedean. Let $w_{1}, w_{2} \in X \otimes Y$ be such that $w_{2}-n w_{1} \in$ $\overline{K_{T}}$ for all $n \in \mathbb{N}$. Since $K_{T}$ is directed, there is a $w_{3} \in K_{T}$ with $w_{3}-w_{2} \in K_{T}$ and $w_{3}+w_{2} \in K_{T}$. Then in $K_{T}$-order we have for all $n$ that

$$
-\frac{1}{n} w_{3} \leq \frac{1}{n} w_{2}=\frac{1}{n} w_{2}-w_{1}-\left(-w_{1}\right)=\frac{1}{n} w_{2} \leq \frac{1}{n} w_{3},
$$

so $\frac{1}{n} w_{2}-w_{1} \rightarrow-w_{1}(\mathrm{ru})$. Hence $-w_{1} \in \overline{K_{T}}$, so $\overline{K_{T}}$ is Archimedean. Thus $\overline{K_{T}} \supseteq K$.

(d) $\Rightarrow$ (b): Let $\left(S, K_{S}\right)$ be a directed Archimedean partially ordered vector space and let $\phi: X \otimes Y \rightarrow S$ be linear and such that $\phi(w) \in K_{S}$ for all $w \in K_{T}$. Let

$$
C:=\left\{w \in X \otimes Y: \phi(w) \in K_{S}\right\}
$$

Then $C \supseteq K_{T}$. We show that $C$ is ru-closed. Let $\left(w_{n}\right)_{n}$ in $C$ and $w \in X \otimes Y$ be such that $w_{n} \rightarrow w(\mathrm{ru})$. Take $a \in K_{T}$ and $\lambda_{n} \rightarrow 0$ such that in $K_{T}$-order we have for all $n$ that $-\lambda_{n} a \leq w_{n}-w \leq \lambda_{n} a$. Then for all $n$ we have

$$
-\lambda_{n} \phi(a) \leq \phi\left(w_{n}-w\right) \leq \lambda_{n} \phi(a)
$$

so $\phi(w) \geq \phi\left(w_{n}\right)-\lambda_{n} \phi(a) \geq-\lambda_{n} \phi(a)$. As $S$ is Archimedean, we get $\phi(w) \in K_{S}$, so $w \in C$. Hence $C$ is ru-closed, so that $K \subseteq C$.

(b) $\Rightarrow$ (a): Let $\left(S, K_{S}\right)$ be a directed Archimedean partially ordered vector space and let $\sigma: X \times Y \rightarrow S$ be a positive bilinear map. Then the induced linear map $\sigma^{*}: X \otimes Y \rightarrow S$ is such that $\sigma^{*}(w) \in K_{S}$ for all $w \in K_{T}$. Hence $\sigma^{*}(w) \in K_{S}$ for all $w \in K$, which means that $\sigma^{*}:(X \otimes Y, K) \rightarrow\left(S, K_{S}\right)$ is positive.

Remark 4.5 Birnbaum [3] and Peressini and Sherbert [12] study the projective cone $K_{T}$ and the biprojective wedge

$$
\begin{aligned}
K_{b}= & \left\{\sum_{i=1}^{n} x_{i} \otimes y_{i}: x_{i} \in X, y_{i} \in Y\right. \\
& \text { such that } \left.\sum_{i=1}^{n} \phi\left(x_{i}\right) \psi\left(y_{i}\right) \geq 0 \forall \phi \in K_{X}^{\prime}, \psi \in K_{Y}^{\prime}, n \in \mathbb{N}\right\},
\end{aligned}
$$

where $K_{X}^{\prime}$ and $K_{Y}^{\prime}$ denote the sets of all positive linear functionals on $\left(X, K_{X}\right)$ and $\left(Y, K_{Y}\right)$, respectively. In some cases $K_{b}$ equals the smallest Archimedean cone containing $K_{T}$. However, Birnbaum gives an example [3, p. 1051] where $K_{b}$ is strictly larger than the smallest Archimedean cone containing $K_{T}$, so that $K_{b}$ is not the Archimedean tensor cone.

It follows from Theorem 4.4 that the Archimedean tensor cone $K$ in $X \otimes Y$ is contained in the cone $K_{F}$ induced by the order of the Fremlin tensor product of the Riesz 
completions of $X$ and $Y$. It is an interesting question whether $K$ and $K_{F}$ coincide. It may also be interesting to relate $K$ and the pseudo-closure of $K_{T}$ in the sense of [8].

Acknowledgments O. van Gaans acknowledges the support by a 'VIDI subsidie' (639.032.510) in the 'Vernieuwingsimpuls' programme of the Netherlands Organisation for Scientific Research (NWO). The authors thank J.J. Grobler whose questions inspired this work.

Open Access This article is distributed under the terms of the Creative Commons Attribution Noncommercial License which permits any noncommercial use, distribution, and reproduction in any medium, provided the original author(s) and source are credited.

\section{References}

1. Aliprantis, C.D., Burkinshaw, O.: Positive Operators. Academic Press Inc., London (1985)

2. Allenby, P.D., Labuschagne, C.C.A.: On the uniform density of $C(X) \otimes C(Y)$ in $C(X \times Y)$. Indag. Mathem., N.S. 20(1), 19-22 (2009)

3. Birnbaum, D.A.: Cones in the tensor product of locally convex lattices. Am. J. Math. 98(4), 10491058 (1976)

4. Buskes, G., van Rooij, A.C.M.: The vector lattice cover of certain partially ordered groups. J. Austral. Math. Soc. (Series A) 54, 352-367 (1993)

5. Buskes, G., van Rooij, A.C.M.: The Archimedean $\ell$-group tensor product. Order 10, 93-102 (1993)

6. Ellis, A.J.: Linear operators in partially ordered normed vector spaces. J. London Math. Soc. 41, 323332 (1966)

7. Fremlin, D.H.: Tensor products of Archimedean vector lattices. Am. J. Math. 94, 777-798 (1972)

8. Grobler, J.J., Labuschagne, C.C.A.: The tensor product of Archimedean ordered vector spaces. Math. Proc. Camb. Philos. Soc. 104, 331-345 (1988)

9. Hulanicki, A., Phelps, R.R.: Some applications of tensor products of partially-ordered linear spaces. J. Funct. Anal. 2, 177-201 (1968)

10. Martinez, J.: Tensor products of partially ordered groups. Pac. J. Math. 41, 771-789 (1972)

11. Merklen, H.: Tensor products of ordered vector spaces. Univ. Nac. Ingen. Inst. Mat. Puras Apl. Notas Mat. 2, 41-57 (1964)

12. Peressini, A.L., Sherbert, D.R.: Ordered topological tensor products. Proc. London Math. Soc. 19(3), 177-190 (1969)

13. van Gaans, O., Kalauch, A.: Tensor Products of Archimedean Partially Ordered Vector Spaces, Report MI-2010-01. Mathematical Institute, Leiden University (2010)

14. van Haandel, M.: Completions in Riesz Space Theory. Ph.D. Thesis. University of Nijmegen (1993) 